\title{
Spatial Correlation Based Clustering with dynamic CH Selection Scheme towards Enhanced QoS Metrics in Wireless Sensor Networks
}

\author{
Panchikattil Susheelkumar S, D. J. Pete
}

\begin{abstract}
Resource constrained wireless sensor nodes are generally randomly distributed in a given area of interest to sense required information and the sensed data is transmitted to the sink station or Base Station (BS) through various clustering and data routing algorithms. The standard clustering algorithms that are aimed at efficient data routing techniques are Low Energy Adaptive Clustering Hierarchy (LEACH) algorithm, Distributed Energy Efficient Clustering (DEEC) algorithm, Stable Election Protocol (SEP) and various others. Most of these algorithms are the various variants of $\mathrm{LEACH}$. Our proposed scheme of data gathering and routing is based on a two hop structure wherein the Cluster Leader (CL) forwards the aggregated received data from the Cluster members to the Sink directly. The Chance-value that determines the Cluster Leader in a round is decided by a combination of parameters specific to the Sensor nodes in the cluster. In our simulation approach we have also tried to analyze the effect of changing density of sensor nodes in the select area. Thus in our proposed scheme, we embark on a fixed Clustering scheme where the $C L$ is selected dynamically so as to extend the network's lifetime and achieve enhanced throughput in comparison to the standard algorithms like DEEC, SEP and LEACH.
\end{abstract}

Keywords : Cluster-Leader, Network Life-Time, Sensing Range, Spatial Correlation.

\section{INTRODUCTION}

Wireless sensor networks have got profound applications in today's world ranging from activity monitoring, weather forecasting, agriculture, home automation to security planning. Since most of these applications are beyond the scope of human interference or accessibility of sensor nodes, the main challenge is to conserve the energy for increased life-time of these networks with limited energy [1,2].

Revised Manuscript Received on February 05, 2020.

* Correspondence Author

Panchikattil Susheelkumar S*, Research Scholar, Department of Electronics Engineering, Datta Meghe College of Engineering, Sector-3, Plot-98, Airoli, Navi Mumbai, Pin: 400708 p_sushilkumar@rediffmail.com

D. J. Pete, Professor \& Head, Department of Electronics Engineering, Datta Meghe College of Engineering, Sector-3, Plot-98, Airoli, Navi Mumbai, Pin: 400708

(C) The Authors. Published by Blue Eyes Intelligence Engineering and Sciences Publication (BEIESP). This is an open access article under the CC BY-NC-ND license (http://creativecommons.org/licenses/by-nc-nd/4.0/)
Hierarchical Clustering approach is normally adopted for energy conservation and Network life-time extensions, wherein the cluster members forward their sensed data to their leader who then aggregates the data received from all its cluster members and forwards the aggregated data to the Sink or BS. LEACH [3-8],

SEP [4,7-10], DEEC [7-9,11] are various implementations of the same approach but are segregated by the differing parameters taken into consideration for cluster formation and its Cluster Head selection or election. Our approach is also based on a hierarchical clustering type depending on a Spatial correlation based clustering and a combined parameter based Cluster Head selection, thus evening out the energy utilization among all cluster nodes and thereby enhancing the network lifetime. Here, in our approach, we have taken advantage of spatial correlation between sensor nodes to effect better clustering.

Further, the paper enlightens us about some related papers in section-II, while section-III \& IV is related to correlation model and energy model adopted in our approach respectively. Section- V \& VI details our proposed methods and algorithms implemented in this paper respectively. Section-VII presents our results and findings in detail while section-VIII details the conclusion along with future scope.

\section{RELATED WORK}

In paper [3,4], the authors have detailed the working of LEACH algorithm and its variants. LEACH represents the foremost protocol under the hierarchical clustering protocols that was developed in order to effect better energy utilization among sensor nodes. But the random selection of Cluster Heads based on a probabilistic manner effected a nonuniform $\mathrm{CH}$ distribution. Also the $\mathrm{CH}$ selection is independent of the residual energy factor or the location factor. These drawbacks resulted in an ineffective performance as far as LEACH protocol is concerned. In paper [9], Zhidong Z. et al. have given a comparative analysis of LEACH, SEP and DEEC algorithm. SEP is basically an heterogeneous LEACH with two types of nodes namely the normal node and the advance nodes. The advance nodes are equipped with higher initial energy than that of normal nodes. Thus by embracing advance nodes into the system, the stability of network is enhanced. But the disadvantage is the requirement of higher initial energy in the system to enhance the stability. S. Singh et al. 
[11] have analyzed DEEC protocol consisting of multi-level initial energy sensor nodes which enhances the network life-time and the stability but at the cost of higher initial energy requirement. In paper [12], data aggregation by the cluster leader employs the correlation between the sensed data by sensor nodes. In paper [13], the nodes with similar readings are grouped together to form clusters based on some correlation between sensor nodes.

The authors have based their distance calculations on Euclidean and Manhattan distance formulae to get node location and similarity. Liu et al. [14] have checked on the Manhattan distance formulae to find dissimilarity in the sensed data of two nodes. Shen et al. have used the tree routing concept and taken forward the similarity concept using Manhattan distance approach in paper [15].

In our proposed approach, we have taken into consideration parameters like sensing range of sensor nodes along with the residual energy of each node combined with the summative distance of member nodes from the $\mathrm{CH}$ in deciding the $\mathrm{CH}$ for each round, which is not considered in the above approaches discussed so far. Few of the papers have touched upon these parameters individually and hence, we are motivated to analyze the effect of these parameters in unison or combination, on the cluster formation and $\mathrm{CH}$ selection. Thus our simulations have showcased better implementation of correlation models effecting better energy conservation and enhanced network life-time.

\section{PROPOSED CORRELATION MODEL}

In our proposed model we have assumed a uniform Sensing range for all the sensor nodes under consideration. each other. We observe that ad the distance $d_{m n}$ decreases of the two nodes. Since the sensing region of any node is a 3-dimensional volume structure, we would be expressing all the regions considered as 3-dimensional volume (Vol).

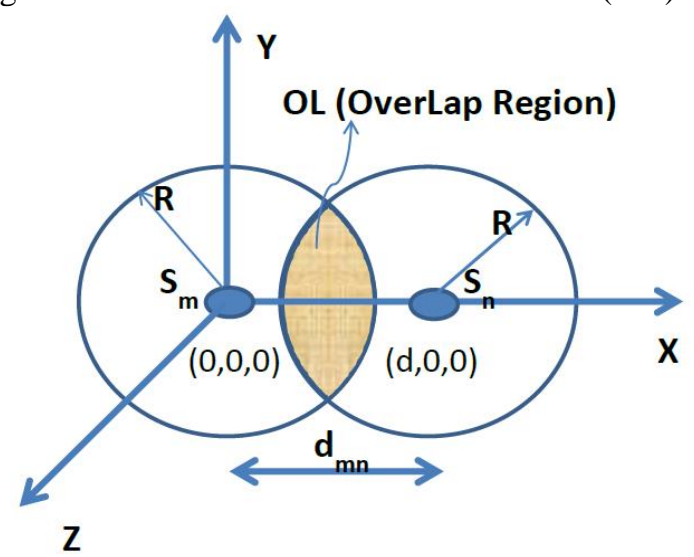

Fig. 1. Over-lapped sensing region of sensor nodes

In the above figure, the overlap region is represented by $\mathrm{Vol}(\mathrm{OL})$ and the correlation between sensors can be expressed as:

$$
\sigma\left(d_{m n}\right)=\frac{\operatorname{Vol}(O L)}{\operatorname{Vol}(\text { Combined })}
$$

From the spherical geometry analysis, the overlap Volume [16] is evaluated as: Let $S_{m}, S_{n}$ be any two sensor node at a distance dmn from below $2 \mathrm{R}$, we see some overlap between the sensing regions

$$
\begin{gathered}
\text { Vol }(O L)=\frac{\prod}{12}(2 R-d)^{2}(d+4 R) \\
\operatorname{Vol}(\text { Combined })=\frac{8 \prod R^{3}}{3}-\frac{\prod}{12}(2 R-d)^{2}(d+4 R) \\
\sigma\left(d_{m n}\right)=\frac{\operatorname{Vol}(O L)}{\operatorname{Vol}(\operatorname{Combined})} \\
=\frac{(2 R-d)^{2}(d+4 R)}{32 R^{3}-(2 R-d)^{2}(d+4 R)}
\end{gathered}
$$

It can be verified that as dmn increases beyond $2 \mathrm{R}, \sigma\left(d_{m n}\right)$ $=0$. Therefore the above expression is valid for $d m n<2 R$ and is expressed as:

$$
\begin{aligned}
& \sigma\left(d_{m n}\right) \\
& =\left\{\begin{array}{cc}
\frac{(2 R-d)^{2}(d+4 R)}{32 R^{3}-(2 R-d)^{2}(d+4 R)} & \text { if } 0 \leq d_{m n}<2 R \\
0 & \text { if } d_{m n} \geq 2 R
\end{array}\right\}
\end{aligned}
$$

Eq.5 represents the desired correlation model. The various symbols used in the above modeling are listed below in table-1 as:

Table- I: Symbols used in the above modelling

\begin{tabular}{|c|c|}
\hline Symbols & Para-meters \\
\hline $\mathrm{R}$ & Sensing Range of nodes: $\mathrm{Sm}, \mathrm{Sn}$ \\
\hline $\mathrm{S}_{\mathrm{m}}$ & $\mathrm{m}^{\text {th }}$ Sensing node \\
\hline $\mathrm{S}_{\mathrm{n}}$ & $\mathrm{n}^{\text {th }}$ Sensing node \\
\hline $\mathrm{Vol}$ & Volume with respect to spheres \\
\hline $\mathrm{OL}$ & Overlap \\
\hline $\mathrm{d}_{\mathrm{mn}}$ & Distance between nodes $\mathrm{S}_{\mathrm{m}}$ and $\mathrm{S}_{\mathrm{n}}$ \\
\hline
\end{tabular}

\section{ENERGY MODEL}

We have adopted the standard energy model referred from paper [3]. The major constituent of energy expended in any Wireless Sensor Network is the propagation energy decided by the second power or fourth power of distance between the transmitter and receiver depending on whether the distance is greater than or less than the crossover distance. The other part of the energy is used for the various electronics involved in the transmission and reception of data. Together, the transmission energy required for transmitting a L - bit message can be expressed as: 


$$
\mathrm{E}_{\mathrm{trxx}}=\mathrm{LE}_{\mathrm{elxx}}+\mathrm{L}^{*} \mathrm{pl} \mathrm{d}_{\mathrm{trxx}}{ }^{(\mathrm{n})}
$$

where $\mathrm{L}$ : total number of bits in any message, $\mathrm{E}_{\mathrm{elxx}}$ : Energy expended on electronics per bit, $\mathrm{pl}^{*} \mathrm{~d}_{\mathrm{trxx}}{ }^{\mathrm{n}}$ : is propagation energy required to cover the transmission distance per bit (here propagation loss dictates the value of $\mathrm{pl}$,

$\mathrm{d}_{\mathrm{trxx}}$ is the distance between the transmitter and receiver and $\mathrm{n}$ represents the propagation loss exponent)

For $\mathrm{d}_{\mathrm{trxx}}<\mathrm{d}_{0}$, 'pl' assumes values dictated by free-space equation. Hence the transmission energy [17], can be concluded as:

$$
E_{\text {trxx }}=L E_{\text {elxx }}+L \epsilon_{\text {fspace }} d_{\text {trxx }}{ }^{2}
$$

where $\epsilon_{\text {fspace }}=\mathrm{pl} \mathrm{represents} \mathrm{free-space} \mathrm{factor}$

And for $\mathrm{d}_{\mathrm{trxx}}>\mathrm{d}_{0}$, we have

$$
E_{\text {trxx } 2}=L E_{\text {elxx }}+L \epsilon_{\text {mpath }} d_{\text {trxx }}{ }^{4}
$$

where 'pl' is specified by the multi-path transmission factor $\epsilon_{\text {mpath. }}$. Here cross-over distance $\mathrm{d}_{0}$ is given by:

$$
d_{0}=\sqrt{\frac{\varepsilon_{\text {fspace }}}{\varepsilon_{\text {mpath }}}}
$$

where $\epsilon_{\text {fspace }}=10$ picoJoules/bit $/$ Sq.metre and $\epsilon_{\text {mpath }}=$ 0.0013 picoJoules/bit/metre ${ }^{4}$ assuming a frequency of 914 k.Hz and a bit rate of $1 \mathrm{Mbps}$ [9].

\section{PROPOSED METHOD}

Here, all the sensor nodes are GPS enabled and the position of each node along with its ID is intimated to the sink at the start of the operation itself. All the sensor nodes are assumed to be energized to the same level at the start. Clusters are finalized based on our predefined spatial correlation criterion at the sink level and the same cluster group is maintained till the exhaustion of network energy. Cluster formation is controlled and aided by the sink. During each round the leader of the cluster group is self-anointed within the group based on a combination of deciding parameters like the residual energy of the member nodes and the location of each cluster member. The algorithm to check the eligibility of a sensor node to become a Leader for the forthcoming round is run at each node independently.

\section{A. Cluster Formation, CH Election \& Data Movement}

Cluster formation is initiated by the sink once in its network lifetime. All the nodes are GPS equipped and the sink is appraised of their location data. We have not considered the impact of the location enabling technology on a comparative scale with existing algorithms since the same technology is adopted by all existing and proposed algorithms. Sink runs a cluster formation algorithm with the node's sensing range and their location as input data to the algorithm. The algorithm checks for overlap between sensor node's sensing region. After executing number of iteration, the algorithm groups together all the sensor nodes having more than a specific range of overlap in their sensing regions.

In our paper, we have varied the overlapping criterion from (6) $10 \%$ to $80 \%$ in multiple steps. Once the clusters are formulated by the sink, the cluster information is fed to each cluster members. This accomplishes the stage one of the operation. The second stage is the selection of Cluster Head by the cluster group. A cluster-head eligibility check algorithm is then executed by each member of the cluster group and finds a chance-value to become the cluster head. This chance-value is broadcasted by the member within the group. If another cluster member finds its chance-value higher than the previously received one, it then broadcasts its value to the group. Lastly, the member with the highest chance-value is accepted by the group members as the cluster-leader. Hence this process of $\mathrm{CH}$ election is dynamic in nature.

Once the leader is elected for a cluster, the data sensed by each cluster member forwards their data to the Cluster Head. Cluster Head aggregates the data and sends the aggregated data to the sink directly.

\section{ALGORITHM}

Cluster Formation Algorithm (Algorithm-1): This algorithm is implemented at the sink side with the following assumptions:

1. Sink or the base station (BS) has all the sensor node locations, IDs and sensing range.

2. A uniform sensing range of radius 5 units has been allotted for all nodes

3. Centrally located Sink with boundless energy is considered. Hence we have not considered the energy required for broadcasting data as an influential parameter for comparison.

4. There are no resource constraints on the sink from the point of view of implementing complex computations or storage requirement is concerned.

Algorithm1:

A. Assign Sensing Range of Sensor nodes $=5$ units for all nodes

B. Compute Sensing Region Overlap between the $1^{\text {st }}$ node (Sm) and $2^{\text {nd }}$ node $(\mathrm{Sn})$

C. Compute the \%Overlap (correlation of sensing region of the two nodes i.e $\sigma\left(d_{m n}\right)$

D. If \%Overlap is greater than the specified value, group the node- 2 with node- 1 and mark node- 2 as grouped with node- 1 and if the condition is not satisfied mark the node-2 as ungrouped..

E. Repeat step B,C \& D with Node- 1 and the $3^{\text {rd }}$ Node and continue till the last node keeping node- 1 as the centre one.

F. Similarly, select the next ungrouped node as the centre node and repeat steps B, C, D, E \& F with other ungrouped nodes.

G. All the nodes are grouped into clusters defined by the spatial correlation between the sensing regions (volume) of the sensor nodes satisfying the specified value. 
Cluster Leader Election Algorithm (Algorithm-2): This algorithm is implemented at the Node end (each node) with the following assumptions:

1. Sensing range of each sensor node is uniform and is taken as 5 units.

2. Cluster members are updated with CM's Location, their ID, number of members in the cluster and the Sink location at the start itself.

3. These nodes are resource constrained as far as energy and other resources are concerned.

Algorithm-2:

A. Assign Sensing Range of Sensor nodes $=5$ units for all nodes

B. Get the residual energy $E_{\text {res }}$ of the Sensor node.

C. Calculate the distances (Euclidean) of other member nodes from oneself.

D. Calculate the summative distance from the above calculated distance.

E. Calculate the Chance-value of each node to be $\mathrm{CH}$ using

$:\left(\left(0.7 * \mathrm{E}_{\mathrm{res}}\right)+0.3 *(1 / \text { sum.dist })^{2}\right) /\left(\mathrm{E}_{\mathrm{res}}+(1 / \text { Sum.dist })^{2}\right)$

F. This value is broadcasted by the individual member to the group. The other members also broad cast their chance-value if its on a higher level than the previous broadcast.

G. The node with the highest valued Chance-value is accepted as the Cluster-Head.

H. The Cluster members then forward their sensed data to the $\mathrm{CH}$ which then aggregates the data received from the CMs.

I. The aggregated data is then forwarded by the $\mathrm{CH}$ to the sink or BS.

J. CH reports the ID of any dead node in its cluster to the Sink.

K. Round ends and then repeat from step-B.

VII. RESULTS AND DISCUSSION

Table- II: Various Parameters taken for simulation (@MATLAB 2016a)

\begin{tabular}{|c|c|c|}
\hline No & Para-meters & Value/Symbol \\
\hline 1 & Total-Nodes & 100 \\
\hline 2 & Field-Area & $100^{*} 100$ \\
\hline 3 & Node Energy at start & $0.5 \mathrm{~J}$ \\
\hline 4 & Sensing Range R & 5 \\
\hline 5 & $\begin{array}{c}\text { Distance between node } \\
\mathrm{S}_{\mathrm{m}} \text { \& } \mathrm{S}_{\mathrm{n}}\end{array}$ & $\sigma\left(\mathrm{d}_{\mathrm{mn}}\right)$ \\
\hline 6. & $\begin{array}{c}\text { Correlation Coefficient } \\
\text { (Free Space factor for } \\
\text { shorter distance }\left(\mathrm{d}<\mathrm{d}_{0}\right)\end{array}$ & $0.0013 \mathrm{pJ} / \mathrm{bit} / \mathrm{m}^{2}$ \\
\hline 7. & $\begin{array}{c}\text { Multi path factor for } \\
\text { longer distance }\left(\mathrm{d}>\mathrm{d}_{0}\right)\end{array}$ \\
\hline 9. & $\begin{array}{c}\text { Energy consumed in the } \\
\text { Electronic circuits to } \\
\text { transmit or receive the } \\
\text { signal }\end{array}$ & $50 \mathrm{~nJ} / \mathrm{bit}$ \\
\hline 10. & $\begin{array}{c}\text { Energy expended for data } \\
\text { aggregation }\end{array}$ & $5 \mathrm{~nJ} / \mathrm{bit}$ \\
\hline 11. & Message Length & $4000 \mathrm{bits}$ \\
\hline
\end{tabular}

We have simulated for various Spatial Correlation Coefficient as listed below:

Table- III: Correlation coefficients or Sensor Region Overlap consideration for cluster formation.

\begin{tabular}{|c|c|c|c|c|c|c|c|c|}
\hline $\begin{array}{c}\text { Parameter } \\
\text { /Condition } \\
\text { No }\end{array}$ & 1 & 2 & 3 & 4 & 5 & 6 & 7 & 8 \\
\hline $\begin{array}{c}\text { Correlation } \\
\text { Coefficient } \\
\sigma\left(d_{m n}\right)\end{array}$ & 0.1 & 0.2 & 0.3 & 0.4 & 0.5 & 0.6 & 0.7 & 0.8 \\
\hline $\begin{array}{c}\text { Over-Lap } \\
\%\end{array}$ & 10 & 20 & 30 & 40 & 50 & 60 & 70 & 80 \\
\hline
\end{tabular}

As a case study, we have tried to study the effect of varying correlation coefficient on the clustering process with two different density node sets (sets of 100 nodes and 200 nodes) spread randomly in an area of $100 * 100 \mathrm{Sq}$. Units in two separate simulation experiments.

Initially, we studied the simulation with 100 sensor nodes and found the impact of the variation of correlation coefficient on the various parameters of importance which is highlighted in table-4. Similarly the impact of correlation coefficient variation with 200 sensor nodes is highlighted in table-5. Graphically all these variations are captured in the graphical figures below from Fig.4 to Fig.11.

Below we are only showing the sensor node distribution and cluster formation for an over-lap percent of $10 \%$ and $40 \%$ for representation purpose as such.

a) Correlation Coefficient -0.1 or Sensing Region Over-lap percent $-10 \%$
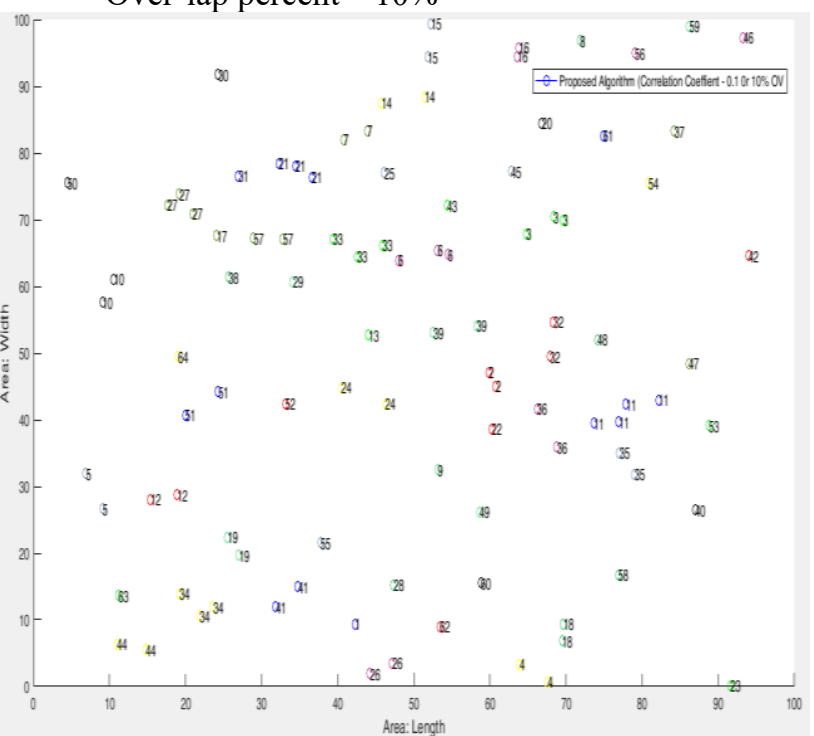

Fig. 2. Sensor node distribution \& cluster formation

$$
(\sigma=0.1)
$$

b) Correlation Coefficient - 0.4 or Sensing Region Over-lap percent $-40 \%$ 


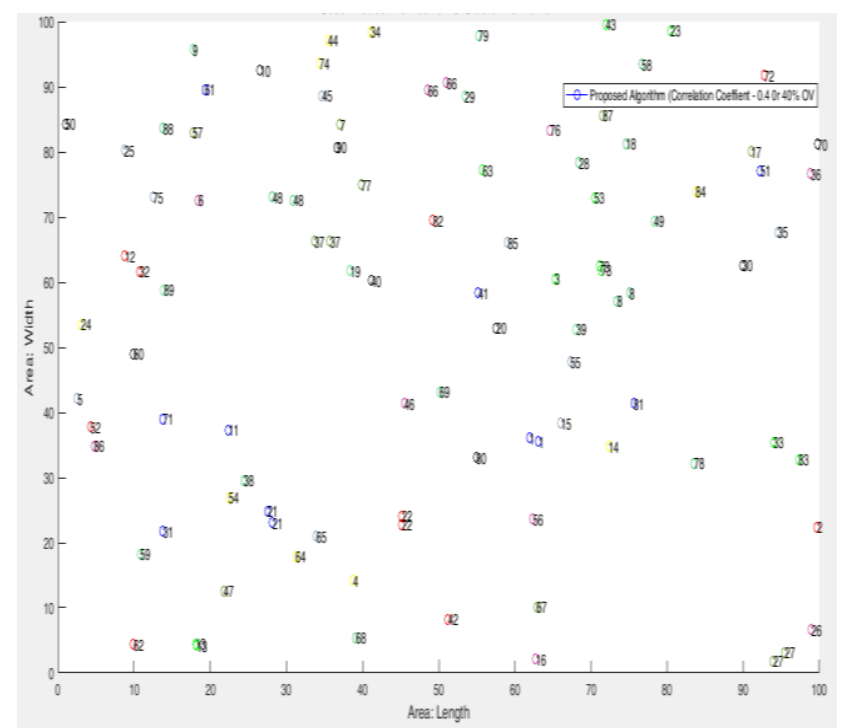

Fig. 3. Sensor node distribution \& cluster formation

$$
(\sigma=0.4)
$$

Simulation results for all the above listed correlation coefficients are shown below for set of 100 sensor nodes (SN) spread in an area of $100 * 100$ Sq. Units:

a) Correlation Coefficient -0.1 or Sensing Region Overlap percent $-10 \%$

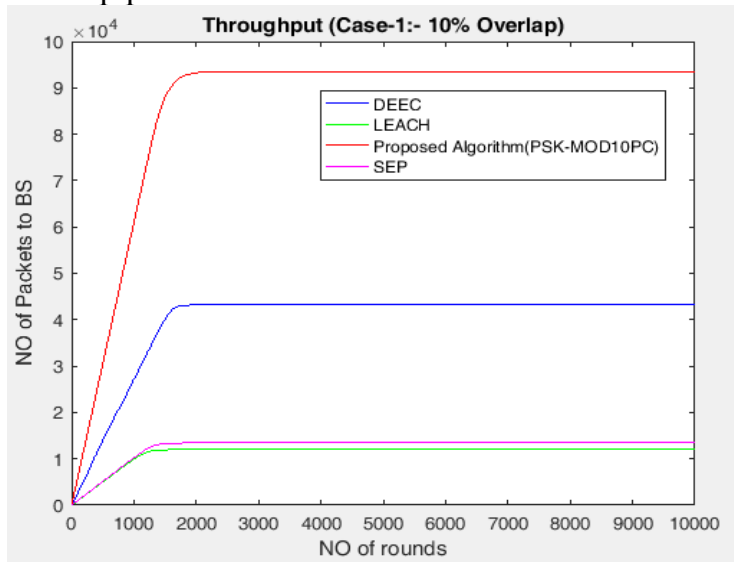

Fig. 4 (a). Throughput $(S N=100 \vdots \sigma=0.1)$

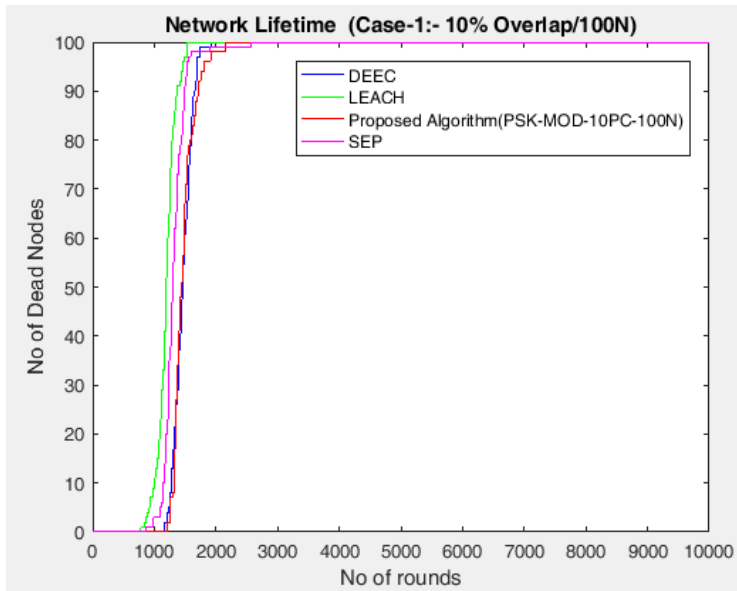

Fig. 4 (b). Network life-time $(S N=100 \vdots \sigma=0.1)$

b) Correlation Coefficient -0.2 or Sensing Region Overlap percent $-20 \%$

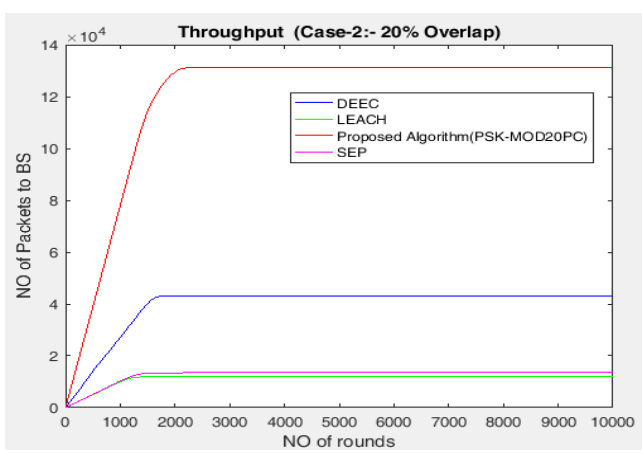

Fig. 5(a). Throughput $(S N=100 \vdots \sigma=0.2)$

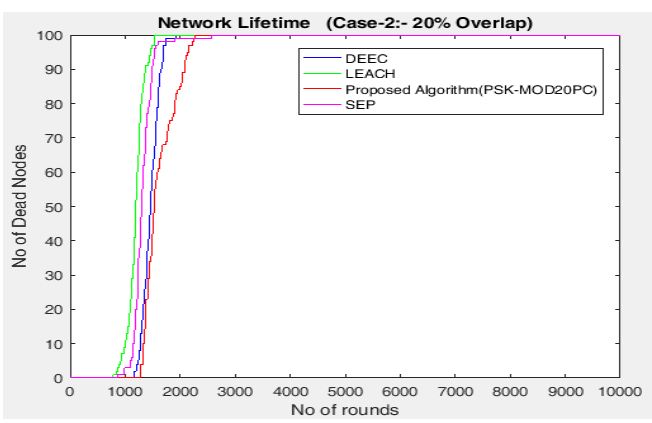

Fig. 5(b). Network lifetime $(S N=100 \vdots \sigma=0.2)$

c) Correlation Coefficient - 0.3 or Sensing Region Overlap percent $-30 \%$

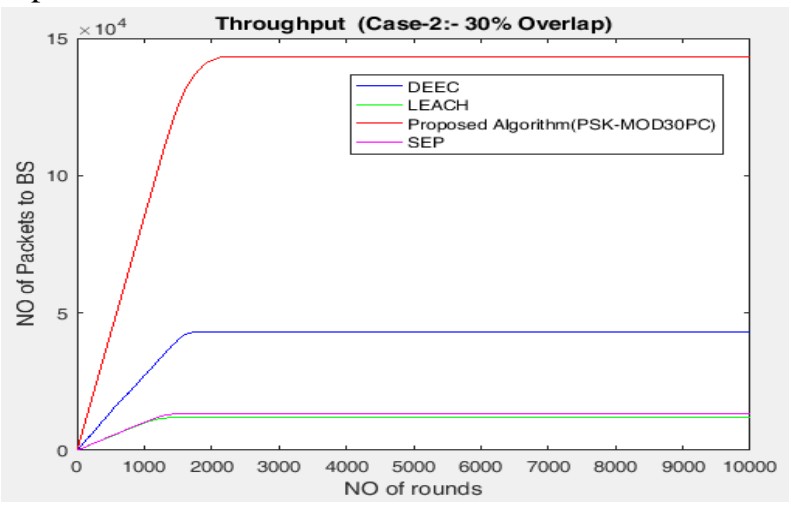

Fig. 6(a). Throughput $(S N=100 \vdots \sigma=0.3)$

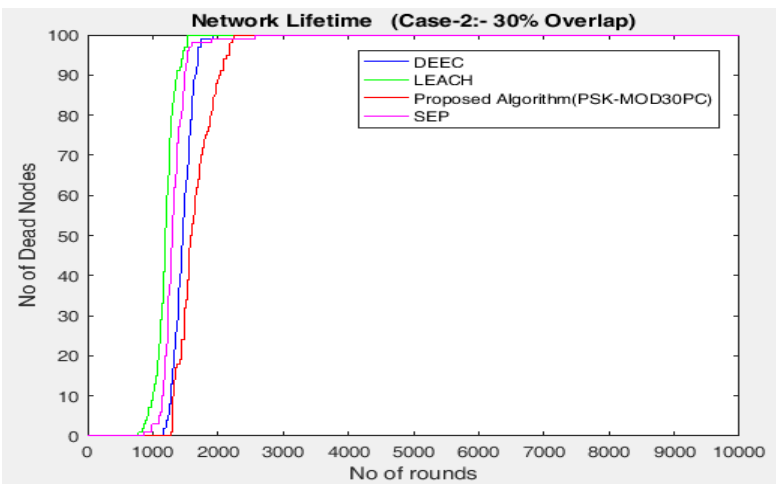

Fig. 6(b). Network life-time $(S N=100 \vdots \sigma=0.3)$

d) Correlation Coefficient - 0.4 or Sensing Region Overlap percent $-40 \%$

Published By:

Blue Eyes Intelligence Engineering

4268 \& Sciences Publication 


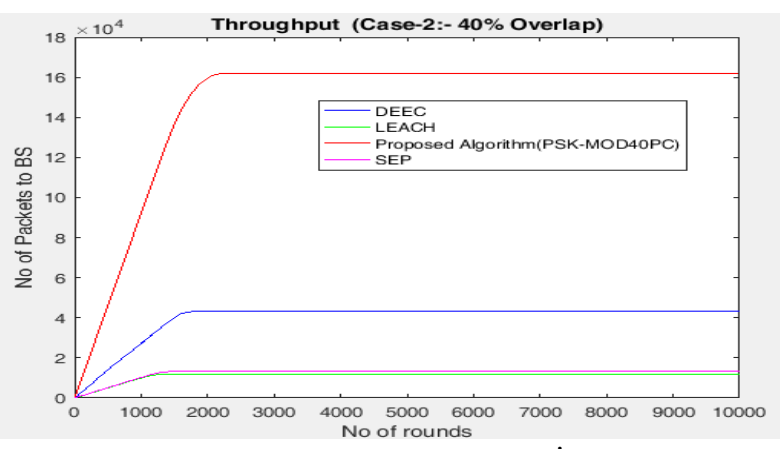

Fig. 7(a). Throughput $(S N=100 \vdots \sigma=0.4)$

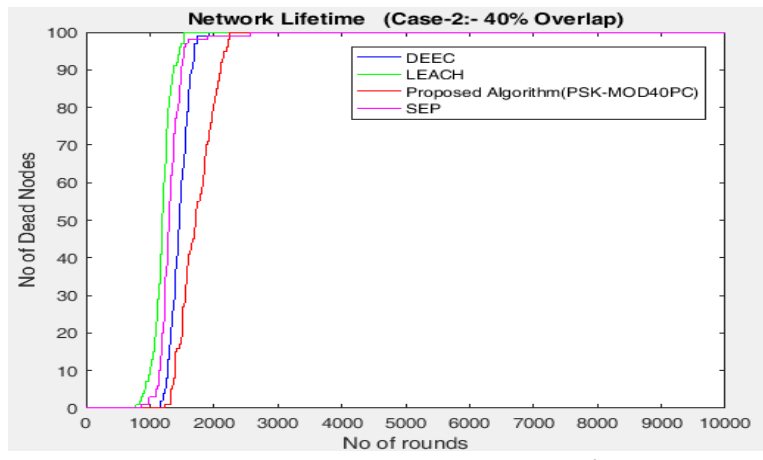

Fig. 7(b). Network lifetime $(S N=100 \vdots \sigma=0.4)$

e) Correlation Coefficient - 0.5 or Sensing Region Overlap percent $-50 \%$

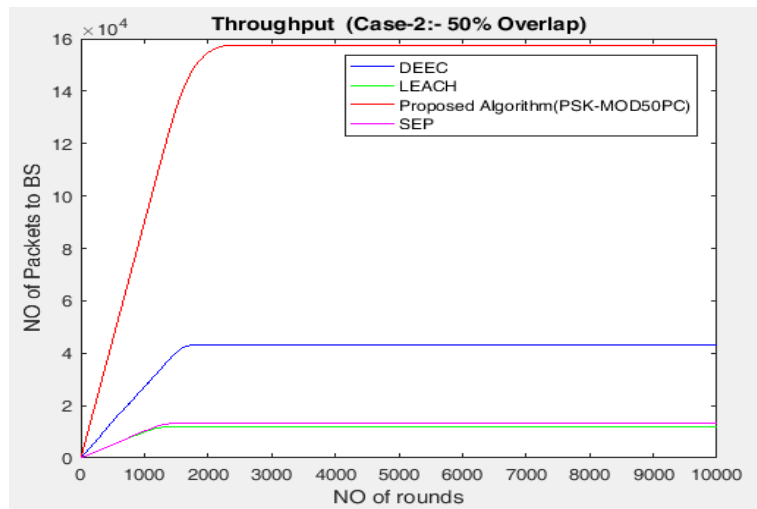

Fig. 8(a). Throughput $(S N=100 \vdots \sigma=0.5)$

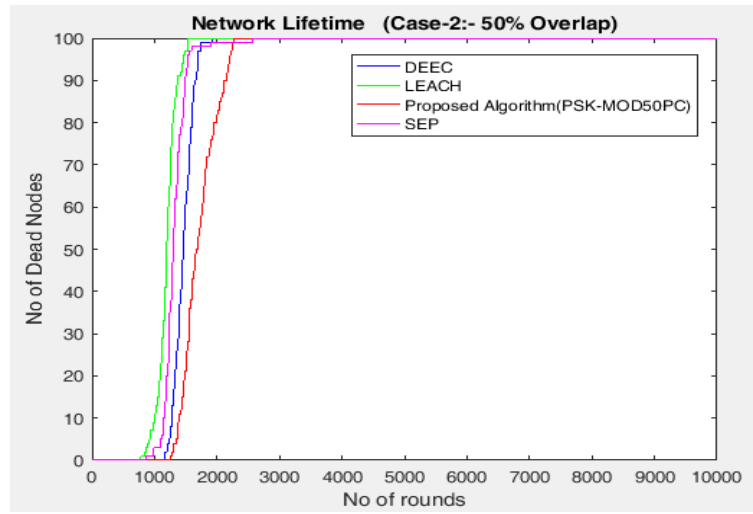

Fig. 8(b). Network lifetime $(S N=100 \vdots \sigma=0.5)$

f) Correlation Coefficient - 0.6 or Sensing Region Overlap percent $-60 \%$

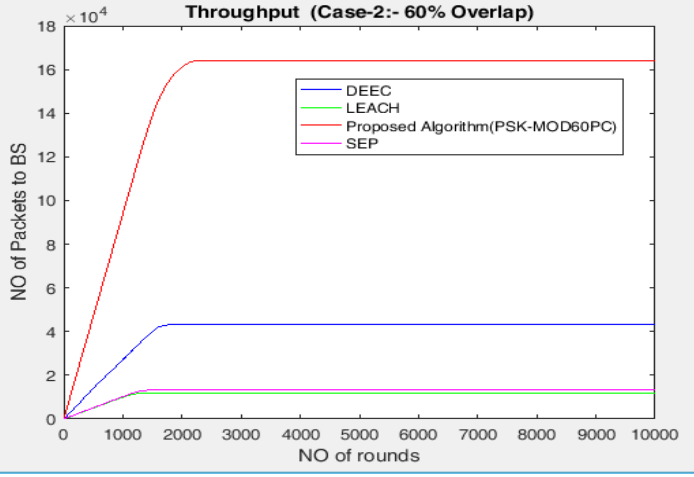

Fig. 9(a). Throughput ${ }^{(S N=100 \vdots \sigma=0.6)}$

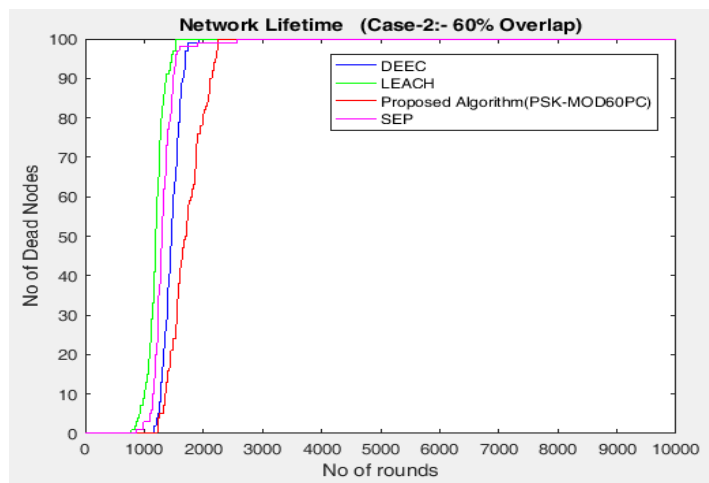

Fig. 9(b). Network lifetime ${ }^{(S N=100 \vdots \sigma=0.6)}$

g) Correlation Coefficient - 0.7 or Sensing Region Overlap percent - 70\%

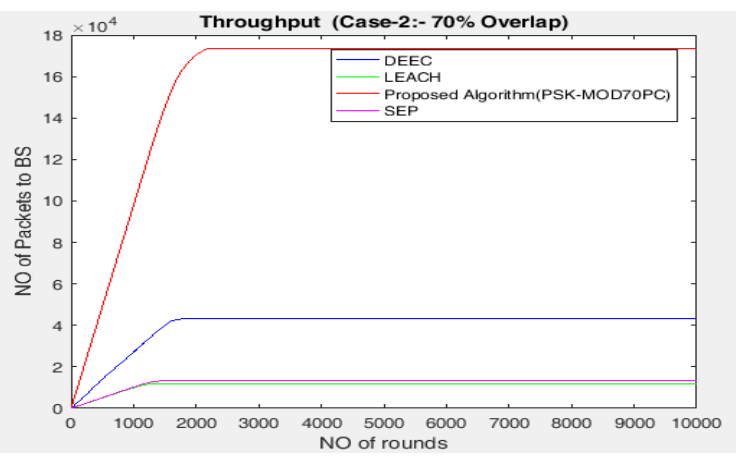

Fig. 10(a). Throughput $(S N=100 \vdots \sigma=0.7)$

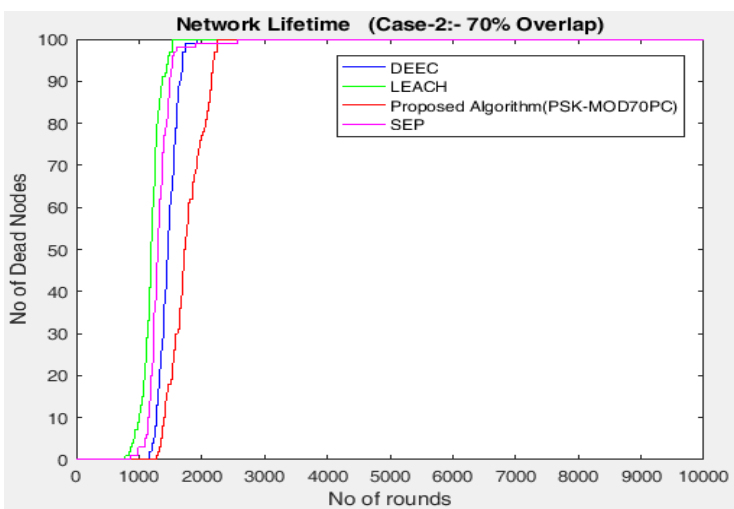

Fig. 10(b). Network lifetime $(S N=100 \vdots \sigma=0.7)$

Published By:

Blue Eyes Intelligence Engineering

DOI: 10.35940/ijeat.C6634.029320

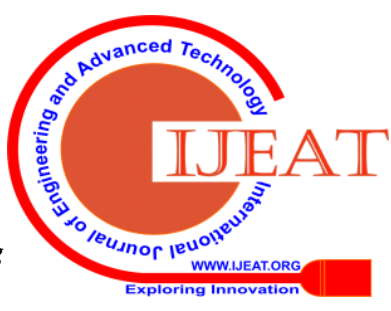


h) Correlation Coefficient - 0.8 or Sensing Region Overlap percent $-80 \%$

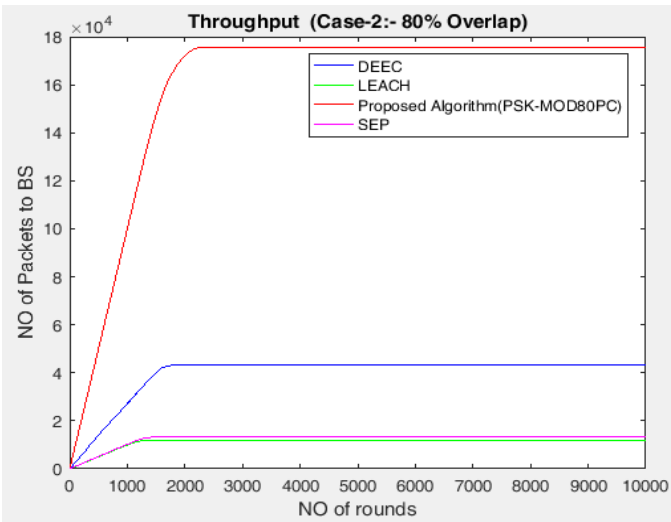

Fig. 11(a). Throughput $(S N=100 \vdots \sigma=0.8)$

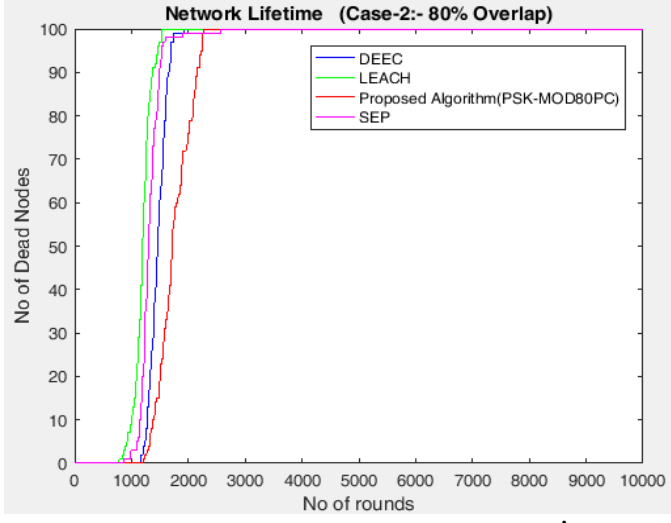

Fig. 11(b). Network lifetime $(S N=100 \vdots \sigma=0.8)$

The detailed results are tabulated below for all the correlation coefficients (or percent over-lap considered).

Table-IV: Correlation coefficients (or Over-lap \%) and corresponding results for 100 Nodes spread across an area of $100 * 100$ Sq. Units

\begin{tabular}{|c|c|c|c|c|c|c|c|c|}
\hline Over-Lap \% & 10 & 20 & 30 & 40 & 50 & 60 & 70 & 80 \\
\hline Number of Clusters formed & 61 & 78 & 85 & 92 & 90 & 94 & 98 & 100 \\
\hline 1st Node Death (ND) Round & 1217 & 1285 & 1275 & 1241 & 1263 & 1222 & 1284 & 1208 \\
\hline 50th ND Round & 1436 & 1524 & 1589 & 1712 & 1678 & 1704 & 1726 & 1712 \\
\hline 75th ND Round (Network LifeTime) & 1532 & 1799 & 1803 & 1943 & 1892 & 1895 & 1960 & 1985 \\
\hline $\begin{array}{c}\text { Throughput [No of Packets sent to Sink in } \\
\text { its Lifetime (75\% ND)] }\end{array}$ & 2161 & 2262 & 2237 & 2253 & 2268 & 2257 & 2258 & 2267 \\
\hline
\end{tabular}

Table- V: Correlation coefficients (Over-lap \%) and corresponding results for 200 Nodes spread across an area of $100 * 100$ Sq. Units

\begin{tabular}{|c|c|c|c|c|c|c|c|c|}
\hline Over-lap \% & 10 & 20 & 30 & 40 & 50 & 60 & 70 & 80 \\
\hline Clusters formed & 97 & 121 & 139 & 159 & 176 & 181 & 193 & 196 \\
\hline 1st (ND) Round & 1203 & 1227 & 1238 & 1235 & 1228 & 1261 & 1228 & 1281 \\
\hline 100th ND Round & 1387 & 1411 & 1471 & 1554 & 1635 & 1691 & 1712 & 1765 \\
\hline $\begin{array}{c}\text { 150th ND Round } \\
\text { (Life-Time) }\end{array}$ & 1645 & 1556 & 2010 & 1810 & 1902 & 1940 & 1985 & 1993 \\
\hline 200th ND Round & 3429 & 2250 & 2267 & 2254 & 2273 & 2268 & 2273 & 2254 \\
\hline $\begin{array}{c}\text { Throughput [No of } \\
\text { Packets sent to Sink in its } \\
\text { Lifetime (75\% ND)] }\end{array}$ & 144426 & 177416 & 217678 & 258339 & 295259 & 310813 & 333785 & 344473 \\
\hline
\end{tabular}

Table- VI: Parameters observed during simulation of LEACH, SEP and DEEC algorithms.

\begin{tabular}{|c|c|c|c|c|c|c|c|}
\hline \multicolumn{2}{|c|}{ Parameters/ Algorithms } & \multicolumn{2}{|c|}{$\begin{array}{c}\text { 100-Sensor Nodes in 100*100 Sq. } \\
\text { Meters }\end{array}$} & \multicolumn{3}{|c|}{$\begin{array}{c}\text { 200-Sensor Nodes in 100*100 Sq. } \\
\text { Meters }\end{array}$} \\
\hline Clusters formed Roundwise & LEACH & SEP & $\begin{array}{c}\text { DEEC } \\
1 \text { to } 18\end{array}$ & $\begin{array}{c}\text { LEACH } \\
\text { SEP }\end{array}$ & \multicolumn{2}{|c|}{ DEEC } \\
\hline $1^{\text {st }}$ Node Death (ND) Round & 783 & 863 & 1165 & 799 & 941 & 1025 \\
\hline 50\% ND Round & 1192 & 1296 & 1456 & 1210 & 1319 & 1415 \\
\hline 75\% ND Round (LifeTime) & 1268 & 1388 & 1564 & 1294 & 1402 & 1525 \\
\hline 100\% ND Round & 1543 & 2565 & 1916 & 2104 & 4875 & 1863 \\
\hline $\begin{array}{c}\text { Throughput [No of Packets sent to } \\
\text { Sink in its Lifetime (75\% ND)] }\end{array}$ & 11688 & 13081 & 41410 & 23809 & 26825 & 52155 \\
\hline
\end{tabular}

From the above results depicted graphically and in tabular format we observe the following:

LEACH and SEP involves relatively smaller number of clusters in comparison to DEEC and our proposed algorithm. Hence clusters in LEACH and SEP have larger number of cluster members. Hence their energy is more utilized for intra cluster communication and hence data packets delivered to sink is comparatively less than DEEC and our proposed algorithm. While comparing DEEC with our proposed algorithm, we can see that our algorithm follows a fixed number of clusters which is finalized initially and the number of clusters is also relatively higher than that in DEEC for all variations of correlation coefficient or percent overlap considered for cluster formation in our proposed algorithm.
In case of lesser density (100 sensor nodes) of sensor nodes, the first node death in our proposed algorithm is reported lately in all conditions of correlation coefficient by around 40 to 120 rounds i.e. an increase of around $4 \%$ to $10 \%$. While in high density (200 sensor nodes), the first node death is reported lately in all conditions of correlation coefficients in comparison with that reported in DEEC by around 175 to 235 round i.e.an increase of around $14 \%$ to $19 \%$ Network lifetime is normally defined subject to the type of application and the purity of data acquired and other relevant factors of importance.

Published By:

Blue Eyes Intelligence Engineering 
Some may define it as the time till: the death of the first node or the death of $50 \%$ of sensor nodes or the death of $75 \%$ of sensor nodes or the death of all nodes in the network. Here for analysis purpose we have defined the network life-time as the time till the death of $75 \%$ of sensor nodes. Further it is observed from the simulation that LEACH and SEP have relatively lesser network lifetime in comparison with DEEC and our proposed algorithm. While comparing our algorithm with DEEC with respect to network lifetime, in case of lower sensor node density (100 nodes) our algorithm outperforms DEEC for all the conditions of correlation coefficient with an increase of around 235 to 400 rounds i.e. an increase of $12 \%$ to $20 \%$ except for the conditional correlation coefficient of 0.1 , where DEEC fares slightly better than our algorithm. In case of higher density sensor nodes (200 nodes), our algorithm outperforms DEEC for all conditions of correlational coefficients with an increase of around 125 to 500 rounds i.e. an increase of around $6 \%$ to $20 \%$ in the network lifetime.

As far as the throughput of the algorithm is concerned, LEACH and SEP lags far behind DEEC and our proposed algorithm since LEACH and SEP expends major part of energy in intra cluster communication and depends more on the data aggregation accuracy. Further comparing our algorithm with DEEC with respect to system throughput, we can say that in case of low density sensor nodes (100 nodes), our algorithm gives a throughput ranging from 88,000 packets to $1,60,000$ packets which is around twice to four times the throughput seen in DEEC.

\section{CONCLUSION}

From the above results and findings mentioned above, we can say that our proposed algorithm outperforms all the three standard algorithms i.e. LEACH, SEP and DEEC in regards with network lifetime and throughput, thereby showcasing the energy efficiency of our proposed algorithm. Further, analyzing the results represented in table-4 \&5, we can say that our proposed algorithm is more suited for densely distributed sensor nodes. It can be seen from table- 4 that as the conditional correlation coefficient (percent overlap for cluster formation) is increased beyond 0.4 , the $50 \%$ node deaths are effected at a much earlier round in comparison to that seen for the lower correlation coefficients in case of lower density (100 nodes) sensor node distribution. Similarly in higher density (200 nodes) sensor node distribution from table-5, it is observed that $100 \%$ node deaths is impacted in around the same round for correlation coefficients of $0.5,0.6$, 0.7 while for the next higher correlation coefficient of 0.8 shows an death of $100 \%$ nodes at a round even below that of others. Thus the results for correlation coefficients ranging from 0.1 to 0.4 (percent overlap from $10 \%$ to $40 \%$ ) would stand more valid and acceptable.

In future-scope, we can work on making the higher range of correlation coefficients more acceptable.

\section{REFERENCES}

1. N. Srivastava, "Challenges of next-generation WSNs and its impact on society", Journal of Telecommunications, 1(1):128-133, 2010

2. Z. Rezaei, S. Mobin inejad, "Energy saving in wireless sensor networks", International Journal of Computer Science and Engineering Survey, 3(1):23-37, Yr:2012.

3. F. Comeaua, Nauman A., "Analysis--of LEACH Energy Para-meters", Elsevier ScienceDirect Procedia Computer Science5(2011)933-938.
4. Yang L., Q. Wu, T. Zhao, Yong T., Fengshan B. and M. Jin, "An Improved Energy-Efficient Routing Protocol for Wireless Sensor Networks", Sensors 2019, 19,4579,doi:10.3390/s19204579

5. Zhe Yang and Abbas Mohammed, "A survey of routing protocols of wireless sensor networks", Tech.Report, Blekinge Inst. of Technology Sweden, Yr:2010.

6. V. Chandrasekaran, "A Review on hier-archical cluster based routing in WSNs", Journal of global research in computer science, no.2,pp.12-16,Yr:2012.

7. Sudeep T., N. Kumar, J.J. Rodrigues, "A systematic review on heterogeneous routing protocols for WSN", Journal of network and computer Appl., vol.53,pp.39-56,Yr:2015.

8. S.Tripti, Brijesh.K., T.Geetam S., "Performance Comparision of LEACH SEP and DEEC Protocol in Wireless Sensor Network", Proceedings of International Journal of Advances in Computer Networks and its security, 2012

9. Zhidong Z., K.Xu, Gu.Hui and L.Hu, “An Energy-Efficient Clustering Routing Protocol for Wireless Sensor Networks Based on AGNES with Balanced Energy Consumption Optimization.", Sensors, 2018,3938;doi:10.3390/s18113938,www.mdpi.com/journal/sensors.

10. Smaragdakis.G, I.Matta, A.Bestavros, "SEP: A stable election protocol for clustered heterogeneous wireless sensor networks", Second int. workshop on sensor and actor network protocols and Appls. (SANPA Yr:2004),vol. 3

11. S. Singh, A. Malik, R. Kumar, "Energy efficient heterogeneous DEEC protocol for en-hancing life-time in WSNs", ScienceDirect/Engineering Science and Technology, an Int.Journal 20(yr:2017)345-353.

12. F. D. Rango, N. Palmieri, S. Ranieri, "The Spatial-Correlation Based Low Energy Aware Clustering (LEACH) in a WSNs", Information and Communication Technologies and Services, Vol.13,No.:4,Yr:2015.Spl-Issue

13. Bhavana, H. T., and Jayanthi K. M. "Spatial- Correlation based ClusteringAlgorithm for Random \& Uniform Topology in WSNs." Int.Journal of Research in Engg. and Tech. (IJRET) 3.6 (2014): P.No. 83 to87.

14. Z. Liu, W. Xing, Bo Zen, Y. Wang, D. Lu, "Distributed Spatial Correlation-based Clustering for Approximate-Data Collection in WSNs", 2013IEEE 27th Int. Conference on Advanced Information Networking and Applications.

15. M. Shen and S. Chen, "Unequal distributed spatial-correlation-based tree clustering for approximate-data collection," Proc. Int. Conference on Soft Computing in Information Communication Technology (SCICT), Atlantis Press, MayYr:2014,pp.93..97

16. Manjeet Singh, Surender Kumar Soni, "Spatial Correlation-Based Clustering in Wireless Sensor Network", International Journal of Engineering and Technology Innovation, vol. 8,no.4,Yr:2018,pp.294..306.

17. Ghalib A. Shah, M. Omer F. and A. B. Dogar, "MR-LEACH: Multi-hop Routing with Low Energy Adaptive Clustering Hierarchy", 2010 Fourth Int. Conference on Sensor Tech. and Applications, DOI:10.1109/SENSORCOMM.Yr:2010.48

\section{AUTHORS PROFILE}

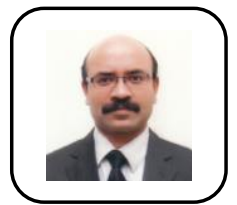

Mr. Panchikattil Susheelkumar Sreedharan, Research Scholar (Ph.D. Electronics Engg.) Department of Electronics Engineering, Datta Meghe College of Engineering, Airoli, Navi-Mumbai. (Affiliated to University of Mumbai). E-mail ID: p_sushilkumar@rediffmail.com

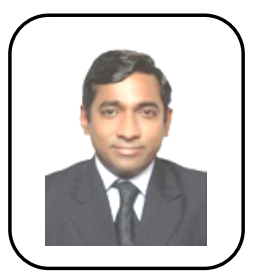

Dr. Dnyandeo J. Pete, (Ph.D. in VLSI Technology from Nagpur University. ,M.Tech. from IIT Bombay), Professor \& Head, Department of Electronics Engineering, Datta Meghe College of Engineering, Airoli, Navi Mumbai. 